\title{
Multipath channel identification by using global optimization in ambiguity function domain
}

\author{
Mehmet Burak Guldogan ${ }^{\mathrm{a}, *}$, Orhan Arikan ${ }^{\mathrm{b}}$ \\ a Department of Electrical Engineering, Linköping University, Linköping SE-58183, Sweden \\ ${ }^{\mathrm{b}}$ Department of Electrical and Electronics Engineering, Bilkent University, Ankara TR-06800, Turkey
}

\section{A R T I C L E I N F O}

\section{Article history:}

Received 16 June 2010

Received in revised form

14 April 2011

Accepted 6 June 2011

Available online 15 June 2011

\section{Keywords:}

Cross ambiguity function (CAF)

Particle swarm optimization (PSO)

Channel identification

Maximum likelihood (ML)

\begin{abstract}
A B S T R A C T
A new transform domain array signal processing technique is proposed for identification of multipath communication channels. The received array element outputs are transformed to delay-Doppler domain by using the cross-ambiguity function (CAF) for efficient exploitation of the delay-Doppler diversity of the multipath components. Clusters of multipath components can be identified by using a simple amplitude thresholding in the delay-Doppler domain. Particle swarm optimization (PSO) can be used to identify parameters of the multipath components in each cluster. The performance of the proposed PSO-CAF technique is compared with the space alternating generalized expectation maximization (SAGE) technique and with a recently proposed PSO based technique at various SNR levels. Simulation results clearly quantify the superior performance of the PSO-CAF technique over the alternative techniques at all practically significant SNR levels.
\end{abstract}

(c) 2011 Elsevier B.V. All rights reserved.

\section{Introduction}

Modern wireless communication systems are designed to operate in multipath environments where the transmitted information arrives at the receiver after reflecting off various obstacles that are present in the environment of the communication. Although at first the presence of multipath arrivals seems to degrade the quality of the communication, a carefully designed communication system can take advantage of the diversity provided by the multipath environment. Diversity in the multipath channels is a result of variation between the directionof-arrivals (DOA), delays and Doppler shifts of the individual channel components. To take full advantage of this diversity, multipath communication channels should be accurately modeled. For this purpose, communication

\footnotetext{
* Corresponding author. Tel.: +4613 282803; fax: +4613139282.

E-mail addresses: bguldogan@gmail.com, bguldogan@isy.liu.se (M.B. Guldogan).
}

systems utilize antenna arrays and sophisticated signal processing techniques to produce estimates for multipath channel parameters. There are a multitude of array signal processing techniques proposed for reliable and accurate estimation for these channel parameters [1]. The maximum likelihood (ML) criterion based channel identification is a commonly used framework due to its superior asymptotic performance. Having determined a parametric signal model, ML estimates are obtained by a search conducted in the parameter space to maximize the likelihood function. The major drawback of the ML technique is its high computational complexity associated with the direct maximization of multimodal and nonlinear likelihood function over a very large dimensional parameter space. Alternative maximization methods are proposed to obtain the ML estimates more efficiently. One of the most popular one to facilitate simple implementation of likelihood function is the expectation maximization (EM) algorithm formulated by Dempster et al. [2]. Simpler maximization steps in lower dimensional parameter spaces are used instead of the original likelihood function. 
Various different forms of the EM algorithm have been developed to further improve the performance. The most popular one is the space alternating generalized EM (SAGE) algorithm, which was developed by Fessler and Hero [3]. In SAGE, parameters are updated sequentially in contrast with the EM where all the parameters are updated simultaneously. Main advantage of the SAGE algorithm over the EM algorithm is its faster convergence resulting in an increased efficiency. Applications of SAGE algorithm are extensively reported in the literature [4-10].

In this paper, a new transform domain array signal processing technique is proposed for identification of multipath communication channels. The received array element outputs are transformed to delay-Doppler domain by using the CAF for efficient exploitation of the delay-Doppler diversity of the multipath signals. In the transform domain, a simple amplitude threshold determined by the noise standard deviation helps to identify the clusters of multipath components. This way, the original channel identification problem is reduced to channel identification problems over the identified path clusters in the delay-Doppler domain and instead of a fitting in time-domain fitting is performed in delayDoppler domain. Since, each cluster has fewer multipath components, there is a significant advantage of conducting the required optimization for identification of channel parameters over the identified clusters. Here, because of its robust performance, we choose to use the PSO to obtain globally optimal values of the channel parameters in each cluster. Since the optimization problem is formulated in the CAF domain of the transmitted signal and the received array outputs, the developed technique is named as the PSO-CAF [11].

Recently, the authors of this work has proposed an alternative technique to incorporate CAF domain information to multipath channel parameter estimation [12,13]. In this technique, which is called as the cross-ambiguity function direction-finding (CAF-DF), the multipath components are tried to be identified one by one in an onion peeling fashion. Then the contribution of the identified path is subtracted from the array outputs and search for another existing component is started on the residual output signals. Unlike the CAF-DF, the proposed PSO-CAF identifies multipath clusters in the delay-Doppler domain and conducts parallel PSO searches on each cluster to estimate parameters of each multipath component resulting in significantly improved estimates especially in denser multipath environments.

The paper is organized as follows. The parametric channel model is detailed in Section 2. In Section 3, maximum-likelihood based parameter estimation is summarized. Basics of PSO is presented in Section 4. Details of the PSO-CAF algorithm is introduced in Section 5. The results of simulation based comparisons of the algorithms are presented in Section 6.

\section{Parametric channel mode}

The proposed PSO-CAF channel identification technique is based on the following commonly used parametric multipath channel model:

$s(t)=\sum_{k=1}^{q} b_{k} p(t-(k-1) T)$,

where $p(t)$ is the transmitted pulse waveform, $q$ is the number of coded pulses, $T$ is the pulse repetition interval in seconds, and $b_{k}$ are \pm 1 . In the following, we will assume that $b_{k}=1,1 \leq k \leq q$. In this way, we will be able to provide our main results with significantly less notational complexity. In a multipath environment, delayed, Doppler shifted and attenuated copies of the transmitted signal impinge on an $M$ element receiver antenna array from different paths. Under the narrowband assumption which is valid when the reciprocal of the bandwidth is much bigger than the propagation of the waveform across the array, output of the antenna array can be modeled as

$\mathbf{x}(t)=\sum_{i=1}^{d} \mathbf{a}\left(\theta_{i}, \phi_{i}\right) \zeta_{i} s\left(t-\tau_{i}\right) e^{j 2 \pi v_{i} t}+\mathbf{n}(t)$,

and in a more compact form

$\mathbf{x}(t)=\mathbf{D}(t, \varphi) \zeta+\mathbf{n}(t)$

where

- $\mathbf{x}(t)=\left[x_{1}(t), \ldots, x_{M}(t)\right]^{T}$ is the array output and [.] $]^{T}$ is the transpose operator,

- $d$ : number of multipaths,

- $\mathbf{a}(\theta, \phi)=\left[a_{1}(\theta, \phi), \ldots, a_{M}(\theta, \phi)\right]^{T}$ is the $M \times 1$ steering vector of the array toward direction of $(\theta, \phi)$,

- $\theta_{i}$ : azimuth angle of the $i$ th path in degrees,

- $\phi_{i}$ : elevation angle of the $i$ th path in degrees,

- $\zeta=\left[\zeta_{i}, \ldots, \zeta_{d}\right]^{T}$ is the $d \times 1$ vector, containing the attenuation and phase terms of individual paths,

- $\tau_{i}$ : time-delay of the $i$ th path,

- $v_{i}$ : Doppler shift of the $i$ th path,

- $\mathbf{n}(t)=\left[n_{1}(t), \ldots, n_{M}(t)\right]^{T}$ is spatially and temporally white circularly symmetric Gaussian noise with variance $\sigma^{2}$,

- channel parameters are collected in the vector $\boldsymbol{\varphi}=$ $\left[\boldsymbol{\varphi}_{1}, \ldots, \boldsymbol{\varphi}_{d}\right]$ and $\boldsymbol{\varphi}_{i}=\left[\tau_{i}, v_{i}, \theta_{i}, \phi_{i}\right]$,

- $\mathbf{D}(t, \boldsymbol{\varphi})=\left[\mathbf{a}\left(\theta_{i}, \phi_{i}\right) s\left(t-\tau_{i}\right) e^{j 2 \pi v_{i} t}, \ldots, \mathbf{a}\left(\theta_{d}, \phi_{d}\right) s\left(t-\tau_{d}\right) e^{j 2 \pi v_{d} t}\right]$ is the $M \times d$ matrix.

Our aim is to estimate the time delays $\tau_{1}, \ldots, \tau_{d}$, Doppler shifts $v_{1}, \ldots, v_{d}$ and DOA's $\left(\theta_{1}, \phi_{1}\right), \ldots,\left(\theta_{d}, \phi_{d}\right)$ of the incoming signals from the $N$ available snapshots of the array output $\mathbf{x}\left(t_{1}\right), \ldots, \mathbf{x}\left(t_{N}\right)$. In this work, we assume that the following conditions hold; the number of paths $d$ is known and the array manifold $\mathbf{a}(\theta, \phi)$ is known. Although we will not go into detail here, there are effective techniques to determine the number of paths [14-17]. Therefore, here we will focus on the details and relative performance of the proposed technique. However, we also propose an alternative PSO-based source number estimation approach in the end of Section 5.

An important performance criterion in multipath channel parameter estimation is the effect of the estimated channel parameters to the performance of the communication receiver system. Given reliable estimates to the channel parameters, the receiver can form the 
following decision signal:

$\hat{\rho}=\int_{0}^{q T} s^{H}(t)\left(\sum_{m=1}^{M} \sum_{i=1}^{d} \hat{\zeta}_{i}^{H} x_{m}\left(t+\hat{\tau}_{i}\right) e^{-j 2 \pi \hat{v}_{i} t} a_{m}^{H}\left(\hat{\theta}_{i}, \hat{\phi}_{i}\right)\right) d t$.

This decision signal is very similar to the decision signal generated by a rake receiver [18]. Here we employed a raking strategy in both delay and Doppler as well as between various DOA's of the multipath components. The following estimate for the SNR of the decision signal given below serves well as a performance criterion between alternative techniques:

$\widehat{\mathrm{SNR}}=\frac{|\hat{\rho}|^{2}}{E_{S} M \sigma^{2} \sum_{i=1}^{d}\left|\hat{\zeta}_{i}\right|^{2}}$,

where $E_{s}$ is the transmitted signal energy.

\section{Maximum-likelihood based parameter estimation}

Maximum likelihood (ML) estimation is a commonly used approach to channel parameter estimation. Assuming that the noise on each pulse transmission is independent, the probability density function of the observations can be obtained as

$P\left[\mathbf{x}\left(t_{1}\right), \ldots, \mathbf{x}\left(t_{N}\right)\right]=\prod_{k=1}^{N} \frac{1}{\left|\pi \sigma^{2} \mathbf{I}\right|} e^{-\left[\left\|\mathbf{e}\left(t_{k}\right)\right\|^{2} / \sigma^{2}\right]}$,

where $|\cdot|$ is for the determinant, $\|\cdot\|$ is for the norm, and $\mathbf{e}\left(t_{k}\right)=\mathbf{x}\left(t_{k}\right)-\sum_{i=1}^{d} \mathbf{a}\left(\theta_{i}, \phi_{i}\right) \zeta_{i} s\left(t_{k}-\tau_{i}\right) e^{j 2 \pi v_{i} t_{k}}=\mathbf{x}\left(t_{k}\right)-\mathbf{D}\left(t_{k}, \boldsymbol{\varphi}\right) \zeta$

The ML estimates that maximize the likelihood function can be written as the maximum of the log-likelihood function:

$[\hat{\boldsymbol{\varphi}}, \hat{\zeta}]=\arg \max _{\boldsymbol{\varphi}, \zeta}\left\{-\mathrm{NM} \log \pi \sigma^{2}-\frac{1}{\sigma^{2}} \sum_{k=1}^{N}\left\|\mathbf{e}\left(t_{k}\right)\right\|^{2}\right\}$,

or equivalently

$[\hat{\boldsymbol{\varphi}}, \hat{\zeta}]=\arg \min _{\boldsymbol{\varphi}, \zeta}\left\{\sum_{k=1}^{N}\left\|\mathbf{e}\left(t_{k}\right)\right\|^{2}\right\}$.

Given the path parameters $\varphi$, path scaling parameters $\zeta$ can be obtained in closed form as

$\hat{\zeta}=\left(\mathbf{D}\left(t_{k}, \boldsymbol{\varphi}\right)^{H} \mathbf{D}\left(t_{k}, \boldsymbol{\varphi}\right)\right)^{-1} \mathbf{D}\left(t_{k}, \boldsymbol{\varphi}\right)^{H} \mathbf{x}\left(t_{k}\right)$,

where $(\cdot)^{H}$ denotes conjugate transpose. Therefore, by substituting (10) into (7), the ML optimization can be reduced to the following optimization problem over the path parameters, $\varphi$, only:

$[\hat{\boldsymbol{\varphi}}]=\arg \min _{\boldsymbol{\varphi}}\left\{\sum_{k=1}^{N}\left\|\mathbf{x}\left(t_{k}\right)-\mathbf{P}_{\mathbf{D}\left(t_{k}, \boldsymbol{\varphi}\right)} \mathbf{x}\left(t_{k}\right)\right\|^{2}\right\}$,

where $\mathbf{P}_{\mathbf{D}\left(t_{k}, \varphi\right)}$ is the projection operator onto the space spanned by the columns of $\mathbf{D}\left(t_{k}, \boldsymbol{\varphi}\right)$ :

$\mathbf{P}_{\mathbf{D}\left(t_{k}, \varphi\right)}=\mathbf{D}\left(t_{k}, \boldsymbol{\varphi}\right)\left(\mathbf{D}\left(t_{k}, \boldsymbol{\varphi}\right)^{H} \mathbf{D}\left(t_{k}, \boldsymbol{\varphi}\right)\right)^{-1} \mathbf{D}\left(t_{k}, \boldsymbol{\varphi}\right)^{H}$.
Table 1

Basic SAGE algorithm.

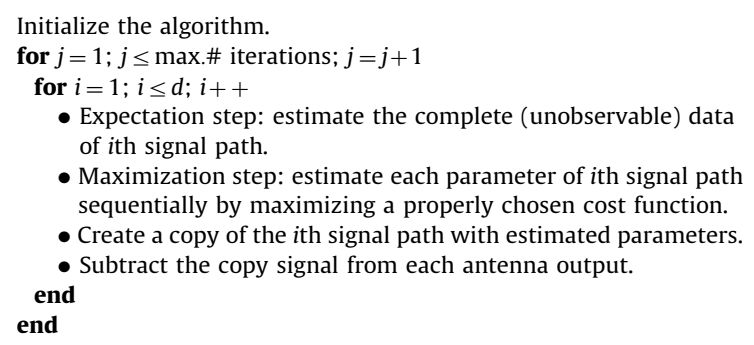

A more compact form of (11) can be given as

$[\hat{\boldsymbol{\varphi}}]=\arg \max _{\boldsymbol{\varphi}}\left\{\sum_{k=1}^{N}\left\|\mathbf{P}_{\mathbf{D}\left(t_{k}, \boldsymbol{\varphi}\right)} \mathbf{x}\left(t_{k}\right)\right\|^{2}\right\}$.

Therefore, one needs to find the global maximum of this $4 \times d$ dimensional optimization problem to identify all four parameters for each path. For large number of multipaths that are common to urban and indoor communication, computational complexity of direct maximization becomes prohibitively high. Moreover, chance of convergence to global optimum point highly decreases due to the overlapped multipath components. One of the most popular approach to obtain more efficient $\mathrm{ML}$ estimates is the EM algorithm [2]. To further improve the speed of convergence of the EM approach, SAGE algorithm has been proposed [3]. In SAGE, parameters are updated sequentially in lower dimensional parameter spaces. In Table 1, the basic form of the SAGE algorithm, which is widely used in channel identification, is presented [7]. Similarly, the SAGE fails to find global solution in searching higher dimensional parameter spaces where there exist overlapped multipath components.

\section{Particle swarm optimization}

Particle swarm optimization (PSO) is an evolutionary stochastic optimization algorithm, developed by Kennedy and Eberhart [19]. PSO has been shown to be very effective in optimizing challenging multidimensional, nonlinear and multimodal problems in a variety of fields such as signal processing [20-23], communication networks [24], biomedical [25,26], control [27,28], robotics [29], power systems [30], electromagnetics [31], image and video analysis [32,33]. It was inspired by the social behavior of animals, specifically the ability of groups of animals to work collectively in finding the desirable positions in a given area. Fish schooling and bird flocking are two very good examples. PSO algorithm operates on a set of solution candidates that are called as swarm of particles. The particles travel through a multidimensional search space, where the position of each particle is adjusted based on a combination of its individual best position and the best position of the whole particle set ever visited. A few key point about PSO should be stated here to clarify the advantages of it over Newton-type techniques: (1) less sensitive to initialization, (2) better 
chance to find global optimum and (3) provides more accurate estimates. Moreover, compared to other global optimization techniques, such as genetic algorithm (GA) [34], some superior properties of PSO can be pointed out that (1) faster in convergence; (2) easier to implement, simpler in concept; (3) can be adapted to different application domains and hybridized with other techniques; (4) interaction between particles is defined in such a way that logic behind the ideal social communication in a community is preserved and diversity of the swarm is maintained through the solution search; (5) better memory management. The components of the PSO setup can be itemized as follows:

- A set of parameters and their corresponding search intervals: For the multipath channel identification, the parameters are the delay, Doppler shift, elevation and azimuth angle of arrivals of each path.

- A fitness function is used to compare the performance of each particle in the swarm: For the multipath channel identification log-likelihood function can be used for this purpose.

- An update strategy for reposition of particles in the swarm.

Although there are variants in the literature, the following stages describe the general dynamics of the PSO.

1. Initialization: Each particle in the swarm starts searching for the optimal position in the solution space at its own random location with a velocity that is random both in its direction and magnitude. This first location is recorded as their personalBest for each particle. globalBest is initialized as the location of the particle that has the best fit.

2. Coordinate update: Each particle travels through the multidimensional search space, where the position and velocity of each particle is adjusted according to certain update rules at each time step. Each particle $l$ consists of three vectors: its location in K-dimensional search space $\mathbf{z}_{l}=\left[z_{l 1}, z_{l 2}, \ldots, z_{l K}\right]$, its historically best position $\mathbf{p}_{l}=\left[p_{l 1}, p_{l 2}, \ldots, p_{l K}\right]$ and its velocity $v_{l}=\left[v_{l 1}\right.$, $\left.v_{l 2}, \ldots, v_{l K}\right]$. In each time step, using the positions of the particle, a fitness function is evaluated. If this fitness value is greater than the value corresponding to personalBest for that particle, or globalBest for the swarm, then these locations are updated with the current location. The velocity and the location of each particle is updated according to the relative positions of personalBest $\left(\mathbf{p}_{i}\right)$ and globalBest $\left(\mathbf{p}_{g}\right)$ by the following equation:

$v_{l k}=\kappa\left(v_{l k}+c_{1} \varepsilon_{1}\left(p_{l k}-z_{l k}\right)+c_{2} \varepsilon_{2}\left(p_{g k}-z_{l k}\right)\right)$,

$z_{l k}=z_{l k}+v_{l k}$,

where $c_{1}$ is so called the cognitive factor that adjusts how much the particle is influenced by the historical best position of his own, $c_{2}$ is so called the social factor that adjusts how much the particle is influenced by the historical best of the swarm, $\varepsilon_{1}$ and $\varepsilon_{2}$ are two uniformly distributed random numbers. $\kappa$ is the constriction factor that balances global and local searches and defined as [35]:

$\kappa=\frac{2}{\left|2-\varsigma-\sqrt{\varsigma^{2}-4 \varsigma}\right|}$,

where $\varsigma=c_{1}+c_{2}$. Recommended values for these constants are $c_{1}=c_{2}=2.05$ and $\kappa=0.72984$.

3. Convergence check: The optimization process is repeated starting at step (2) until convergence is established or the maximum allowed number of iterations are reached.

\section{Proposed PSO-CAF technique}

In a multipath environment, the receiver array output signals are delayed, Doppler-shifted and scaled versions of the transmitted signal. As mentioned in Section 3, formulating a likelihood function for the channel estimation problem is a very common way to extract the signal parameters. However, when the number of paths increases, the ML approach face significant challenges in finding the global maximum of the likelihood function. This is mainly because of the fact that likelihood maximization is performed in time domain, where there is a considerable overlap between the signals received from different paths. Therefore, it is desirable to formulate an alternative optimization problem other than the time domain where the multipath signal components are localized reducing the significant overlapping of components in the time domain. Since typical communication signals are phase or frequency modulated, with large time-bandwidth products, as in radar detection their CAFs are highly localized in the delay-Doppler domain. Therefore, the transformation of the array signal outputs to the CAF domain localizes different multipath signals in clusters to their respective delay and Doppler cell. Although there exist several different representation, symmetrical version of the CAF between the transmitted signal $s(t)$ and the received signal $x(t)$ can be written as [36-38]:

$\chi_{x(t), s(t)}(\tau, v)=\int_{-\infty}^{\infty} x\left(t+\frac{\tau}{2}\right) s^{H}\left(t-\frac{\tau}{2}\right) e^{-j 2 \pi v t} d t$

In the SNR sense, matched filtering is the optimum solution for detection. When the Doppler shift is not known, performance of the receiver that makes use of a matched filter that matched to the transmitted signal may significantly degrade. The CAF characterizes the output of a matched filter when the input signal is delayed and Doppler shifted. In the case of one multipath component, CAF calculation is the optimal solution for detection. If there exist two or more multipath components separated enough in delay Doppler domain, again, CAF surface offers a very useful detection surface by using properly chosen waveforms for the application of interest [37,39-42]. When the phase of each impinging signal on the array is unknown, to detect the multipath clusters on the delay-Doppler domain, absolute values of CAFs at the output of each antenna are calculated and incoherently integrated as:

$\boldsymbol{\chi}(\tau, v)=\frac{1}{M} \sum_{m=1}^{M}\left|\boldsymbol{\chi}_{x_{m}, s(t)}\right|$. 
Since the antennas in the array are closely spaced, peak locations of the CAFs will be nearly the same for each antenna. This means that, after incoherent integration, desired highest peak power on the incoherently integrated CAF surface is not changed notably but noise power is reduced all around the highest peak. In another words, compared to detecting highest peak on one CAF surface, same probability of detection can be obtained with less SNR if detection of the highest point is done on the incoherently integrated CAF surface. Detection performance of the cluster locations that exceeds the detection threshold is improved by this way. This practical approach is widely used in radar signal processing and there are excellent references presenting details of the procedure [37,40].

One important principle that should be mentioned at this point is that the uncertainty principle [37]. Briefly, it says that, if one narrow the peak on AF surface to increase the estimator's local accuracy, the volume removed will reappear somewhere away from the peak and will decrease the estimator's global accuracy. This behavior of the AF indicates that there have to be trade-offs made among the resolution, accuracy, and ambiguity. Therefore, type of the waveform determines the accuracy in resolving multipath components and should be chosen based on the interested problem. Maybe the most famous family of phase codes are the Barker codes [37,43,44]. In this paper, as a transmitted signal, $s(t)$, Barker-13 phase coded pulse train is used, which provided us good delay and Doppler resolution for the interested area on the CAF surface. Barker-13 phase coded waveforms yield a peakto-peak side-lobe ratio of 13 and their CAFs are very localized enabling accurate multipath cluster detection.

Recent multipath channel measurement results show that multipath components are distributed in as clusters within a defined channel spread and impinge onto a receiver in clusters [5,45-47]. In [45], a statistical model, which is based on only temporal clustering structure they observed in their indoor multipath data, is presented. The clusters and the rays/multipaths within the clusters are observed to have Poisson arrival processes with different rates. The clusters are attenuated in amplitude and path arrivals within a single cluster also have amplitudes decayed with patterns that are exponential with time and are parameterized with two time constants; the cluster arrival decay time constant and the ray/multipath arrival decay time constant. A space-time statistical model is presented as an extension of the time-only model of [45] in [46]. In [47], authors make use of the recent advances in the theory of compressed sensing, to formalize the notion of clustered multipath sparsity and to exploit delay, Doppler and spatial diversity. With the motivation from these works, starting point of the proposed PSO-CAF algorithm is the detection of multipath clusters on ambiguity surface. Detection threshold selection procedure includes a trade-off between missed detections and false detections. The threshold should be high enough not to have too large false alarm probability. The threshold should be low enough not to have too small probability of detection. With the usage of previously stated phase coded waveforms the CAF surface provides very localized, peaky and compressed structures that make thresholding a good estimation procedure. By choosing a proper threshold level, multipath cluster detection on delay-Doppler domain can be accurately accomplished. For this purpose a constant false alarm criterion rate (CFAR) based adaptive threshold can be set. Such a strategy is commonly employed by radar target detection [48]. In this paper, first, we find the peak point of the incoherently integrated CAF, $|\chi(\tau, v)|$ and compare it with the noise level. Noise level on the incoherently integrated CAF is quantified with the median operator, since it is relatively insensitive to possible outliers. Specifically, ratio between maximum and median values of the $|\boldsymbol{\chi}(\tau, v)|$ is computed and compared with a properly chosen threshold value. If the calculated ratio is higher than the determined threshold value then that peak point is considered as the location of the multipath cluster $[42,49,50]$.Once the peak location of the multipath cluster is detected, a window of size, $1.5 \Delta \tau \times 1.5 \Delta v$, around the detected peak is determined and PSO optimization is conducted on the extracted data to estimate parameters of each multipath component. Here, $\Delta \tau$ and $\Delta v$ are delay and Doppler resolutions, respectively. Extracted data is the vectorized form of the detected delay-Doppler patch. Therefore, in order to estimate parameters of each multipath component that is in the detected cluster, PSO is used to conduct fitting in delay-Doppler domain. Having estimated the parameters of each multipath component in the cluster, effect of the cluster is eliminated from the array outputs to recurse on the residual for detection of the remaining multipath clusters. This iterative approach is highly efficient and accurate. Note that the elimination of a multipath cluster from the array outputs eliminates both its main and sidelobes from the CAF domain. Thus, weaker multipath clusters that are buried under the sidelobes of the detected and eliminated multipath cluster might become detectable as well. This detection and elimination process is repeated until there is no peak exceeding the detection threshold on the CAF domain. Although detection threshold selection is a common problem for many estimators, once more it is good to stress that; if a high detection threshold is chosen, then the remaining clusters, after successive cancellation procedure, might not be detected. Then, multipath components in that cluster will be underestimated. If a low detection threshold is chosen, then the algorithm will fit to noise and there will be overestimated multipath components. However, since the original time-domain channel identification problem is reduced to channel identification problems over the identified isolated and separated path clusters in the delayDoppler domain by the proposed technique, performance will not effected significantly due to overestimated cluster multipaths.

To illustrate this procedure, consider a synthetic multipath channel with six distinct paths. As shown in Fig. 1(a), the individual multipath signals overlap significantly in time at the output of an array element. However, as shown in Fig. 1(b), the CAF given in (16) between the received signal and the transmitted signal localizes the contribution of different path components in delay-Doppler domain. Moreover, to present the effective localization of the multipath ionospheric reflections on delay-Doppler domain, the CAF surface of a real high-latitude ionospheric 

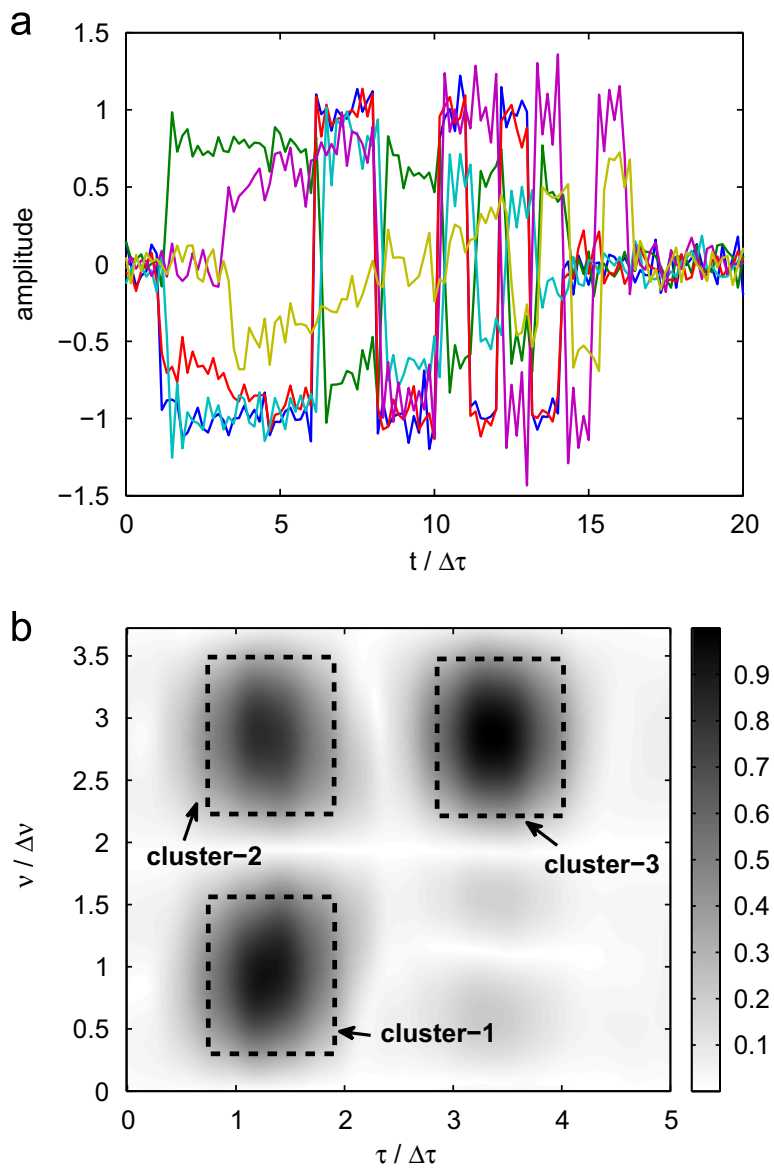

Fig. 1. Barker-13 coded six paths (a) in time domain and (b) in delayDoppler domain localized in three clusters each of which has two paths.

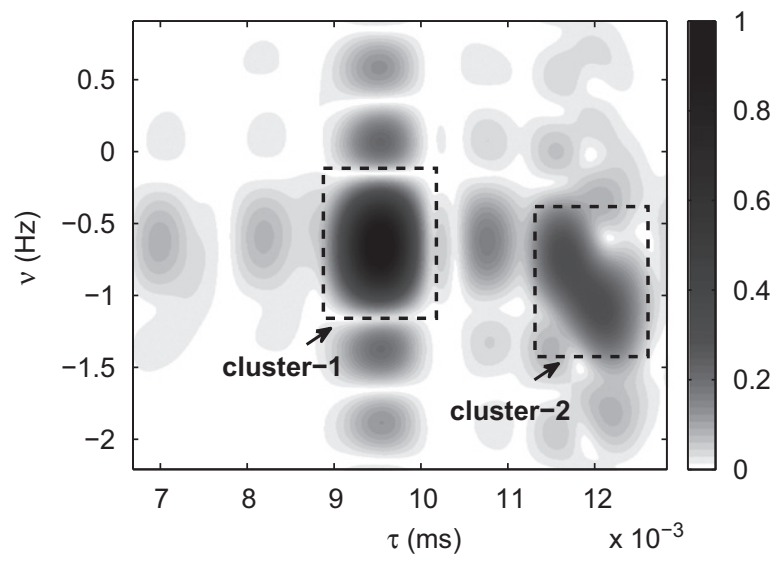

Fig. 2. CAF between recorded multipath high-latitude ionosphere data and the transmitted signal. One dominant reflection in cluster-1 at $\tau=9.5 \mathrm{~ms}$. Two reflections in cluster- 2 between $\tau=11.5$ and $12.5 \mathrm{~ms}$.

communication channel is shown in Fig. 2. As seen from the result, two clusters can be reliably detected. Further analysis on the data has revealed that one of the clusters has a single component and the other has two multipath components $[12,51]$. There is one dominant reflection in cluster- 1 at $\tau=9.5 \mathrm{~ms}$, and there are two reflections in cluster-2 between $\tau=11.5$ and $12.5 \mathrm{~ms}$. This localization enables us to reformulate the channel identification problem as a set of loosely coupled optimization problems in lower dimensional parameter spaces.

Signal flow diagram of the PSO-CAF algorithm is presented in Figs. 3 and 4. $C$ clusters of multipath components present on delay-Doppler domain and the number of multipaths in cluster $c$ is $d_{c}$ for $1 \leq c \leq C$. For example as shown in Fig. 1, six paths are localized in $C=3$ clusters and each cluster consists of two paths. Having identified the location of each cluster, instead of conducting a fitting in time domain, individual PSO searches are conducted on vectorized delay-Doppler patch for estimation of parameters of multipaths in each cluster. Following PSO searches in each cluster, effects of the estimated multipath components are eliminated for a better estimation in the remaining clusters. Since, optimization in each cluster has to be performed multiple times, PSO iterations in each cluster need not to be pursued until convergence is established. Therefore, by cycling over the identified set of clusters, the PSO-CAF technique iteratively provides estimates for each path in each cluster computationally efficiently. In the following, details of the CAF domain optimization for each cluster are presented.

The optimization problem associated with the $c$ th cluster makes use of the following fitness function:

$f_{c}\left(\boldsymbol{\varphi}\left(\mathbf{S}_{c}, \eta\right), \boldsymbol{\zeta}_{c}(\eta)\right)=\sum_{m=1}^{M}\left\|\boldsymbol{\Gamma}_{c, m}-\operatorname{vec}\left(\mathbf{W}_{c} \boldsymbol{\chi}_{\hat{u}_{m}\left(t ; \boldsymbol{\varphi}\left(\mathbf{S}_{c}, \eta\right)\right), s(t)}(\tau, v)\right)\right\|^{2}$,

where $\mathbf{S}_{c}$ is the set containing indexes of $d_{c}$ path components in the $c$ th cluster, $\operatorname{vec}($.$) is vector operator stacking$ the columns of a matrix into a single column vector, $\mathbf{W}_{c}$ is the identifier mask for the $c$ th cluster that selects the delay-Doppler patch that will be used in PSO, and $\boldsymbol{\Gamma}_{c}=$ $\left[\boldsymbol{\Gamma}_{c, 1}, \ldots, \boldsymbol{\Gamma}_{c, M}\right]^{T}$ is the matrix of the $c$ th cluster delayDoppler patch for $M$ antennas with elements:

$\boldsymbol{\Gamma}_{c, m}=\boldsymbol{v e c}\left(\mathbf{W}_{c} \boldsymbol{\chi}_{\hat{y}_{c, m}(t ; \eta), s(t)}(\tau, v)\right)$.

$\chi_{\hat{y}_{c, m}(t ; \eta), s(t)}(\tau, v)$ is the CAF between $\hat{y}_{c, m}(t ; \eta)$ and $s(t)$, $\boldsymbol{\chi}_{\hat{u}_{m}\left(t ; \boldsymbol{\varphi}\left(\mathbf{S}_{c}, \eta\right)\right), s(t)}(\tau, v)$ is the CAF between $\hat{u}_{m}\left(t ; \boldsymbol{\varphi}\left(\mathbf{S}_{c}, \eta\right)\right)$ and $s(t), \hat{\mathbf{y}}_{c}(t ; \eta)=\left[\hat{y}_{c, 1}(t ; \eta), \ldots, \hat{y}_{c, M}(t ; \eta)\right]$ is the estimated array output at the $\eta$ th iteration corresponding to $c$ th cluster:

$\hat{\mathbf{y}}_{c}(t ; \eta)=\mathbf{x}(t)-\sum_{\gamma=1, \gamma \neq c}^{C} \hat{\mathbf{u}}\left(t ; \boldsymbol{\varphi}\left(\mathbf{S}_{\gamma}, \eta\right)\right)$,

where $\hat{\mathbf{u}}\left(t ; \boldsymbol{\varphi}\left(\mathbf{S}_{c}, \eta\right)\right)=\left[\hat{u}_{1}\left(t ; \boldsymbol{\varphi}\left(\mathbf{S}_{c}, \eta\right)\right), \ldots, \hat{u}_{M}\left(t ; \boldsymbol{\varphi}\left(\mathbf{S}_{c}, \eta\right)\right)\right]^{T}$ is the matrix generated with the $c$ th cluster estimated multipath parameters for $M$ antennas with elements:

$\hat{\mathbf{u}}\left(t ; \boldsymbol{\varphi}\left(\mathbf{S}_{c}, \eta\right)\right)=\sum_{i \in \mathbf{S}_{c}} \zeta_{i}(\eta) s\left(t-\hat{\tau}_{i}(\eta)\right) e^{j 2 \pi \hat{v}_{i}(\eta) t} \mathbf{a}\left(\hat{\theta}_{i}(\eta), \hat{\phi}_{i}(\eta)\right)$

In the first iteration, $\eta=1$, for the first cluster, $\hat{\mathbf{y}}_{c}(t ; \eta)$ is initialized as $\hat{\mathbf{y}}_{c}(t ; \eta)=\mathbf{x}(t)$. Using $(21), \chi_{\hat{u}_{m}\left(t ; \varphi\left(\mathbf{S}_{c}, \eta\right)\right), s(t)}(\tau, v)$ can be written as

$\boldsymbol{\chi}_{\hat{u}_{m}\left(t ; \boldsymbol{\varphi}\left(\mathbf{S}_{c}, \eta\right)\right), s(t)}(\tau, v)=\sum_{i \in \mathbf{S}_{c}} \zeta_{i}(\eta) \hat{\mathbf{A}}_{m}\left(\tau, v ; \hat{\boldsymbol{\varphi}}_{i}(\eta)\right)$, 


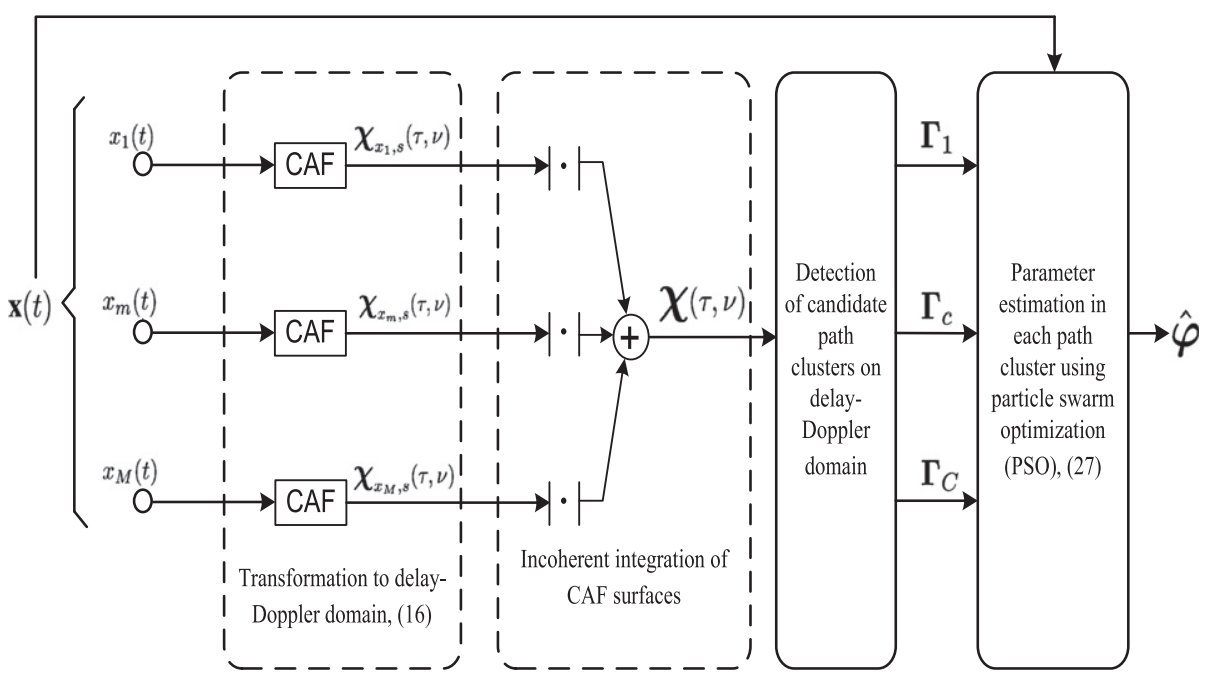

Fig. 3. Signal flow diagram of the PSO-CAF algorithm.

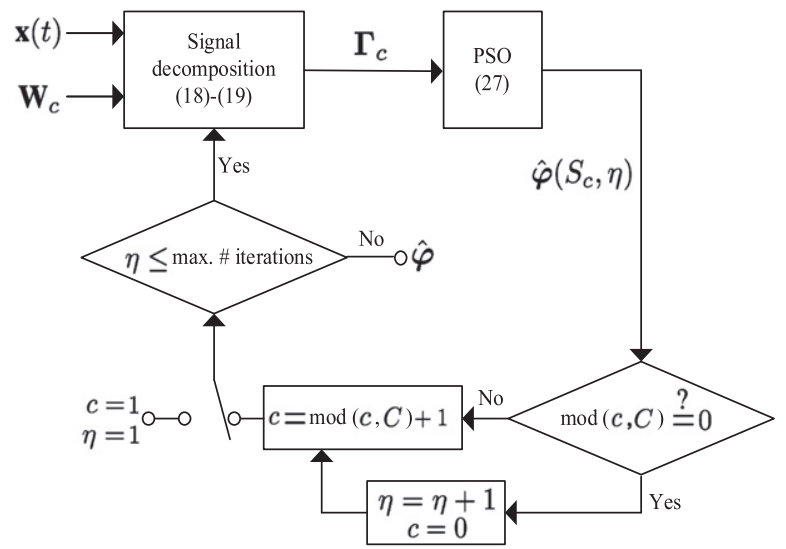

Fig. 4. Signal flow sub-block diagram of the parameter estimation in each cluster using PSO block in Fig. 3.

where $\hat{\mathbf{A}}_{m}\left(\tau, v ; \hat{\boldsymbol{\varphi}}_{i}(\eta)\right)$ is defined as:

$$
\begin{aligned}
\hat{\mathbf{A}}_{m}\left(\tau, v ; \hat{\boldsymbol{\varphi}}_{i}(\eta)\right)= & a_{m}\left(\hat{\theta}_{i}(\eta), \hat{\phi}_{i}(\eta)\right) \int_{-\infty}^{\infty} s\left(t-\hat{\tau}_{i}(\eta)+\frac{\tau}{2}\right) \\
& \times s^{H}\left(t-\frac{\tau}{2}\right) e^{-j 2 \pi\left(v-\hat{v}_{i}(\eta)\right) t} d t
\end{aligned}
$$

By using (19) and (23), a more compact form for the fitness function in (18) can be obtained as

$f_{c}\left(\boldsymbol{\varphi}\left(\mathbf{S}_{c}\right), \zeta_{c}\right)=\sum_{m=1}^{M}\left\|\boldsymbol{\Gamma}_{c, m}-\boldsymbol{Y}_{c, m} \boldsymbol{\zeta}_{c}\right\|^{2}$.

Here, estimate of matrix $\boldsymbol{r}_{c, m}$ is defined as

$$
\hat{\boldsymbol{Y}}_{c, m}=\left[\operatorname{vec}\left(\mathbf{W}_{c} \hat{\mathbf{A}}_{m}\left(\tau, v ; \hat{\boldsymbol{\varphi}}_{\varrho_{1}}(\eta)\right)\right), \ldots, \operatorname{vec}\left(\mathbf{W}_{c} \hat{\mathbf{A}}_{m}\left(\tau, v ; \hat{\boldsymbol{\varphi}}_{\varrho_{d_{c}}}(\eta)\right)\right)\right],
$$

where $\varrho_{1}$ is the first index element of the index set $\mathbf{S}_{c}$ and each column corresponds to a multipath component in the $c$ th cluster. Straightforward minimization with respect to the scale variables $\zeta$ yields

$$
\hat{\zeta}_{c}(\eta)=\frac{1}{M} \sum_{m=1}^{M}\left(\hat{\boldsymbol{Y}}_{c, m}^{H} \hat{\boldsymbol{\Gamma}}_{c, m}\right)^{-1} \hat{\boldsymbol{\Gamma}}_{c, m}^{H} \boldsymbol{\Gamma}_{c, m},
$$

which, as in the ML approach culminating with (11), when substituted into (24), reduces the fitness function for the $c$ th cluster to:

$f_{c}\left(\boldsymbol{\varphi}\left(\mathbf{S}_{c}, \eta\right)\right)=\sum_{m=1}^{M}\left\|\boldsymbol{\Gamma}_{c, m}-\boldsymbol{Y}_{c, m} \hat{\zeta}_{c}(\eta)\right\|^{2}$

Thus, the channel parameter estimates for the $c$ th cluster at $\eta$ th iteration are obtained by minimizing the following optimization problem:

$\hat{\boldsymbol{\varphi}}\left(S_{c}, \eta\right)=\arg \min _{\varphi} f_{c}\left(\boldsymbol{\varphi}\left(\mathbf{S}_{c}, \eta\right)\right)$.

Location of each particle $\mathbf{z}_{l}=\left[\boldsymbol{\varphi}_{\varrho_{1}}, \ldots, \varphi_{\varrho_{i}}, \ldots, \varphi_{\varrho_{d_{c}}}\right]$ in the $K=4 \times d_{c}$ dimensional search space is a solution candidate. The size of the target delay-Doppler patch, $\boldsymbol{\Gamma}_{c}$, determined by the identifier mask $\mathbf{W}_{c}$, is chosen to be $1.5 \Delta \tau \times 1.5 \Delta v$ around the detected peak for the $c$ th cluster. Resolution of delay and Doppler in the CAF domain are $\Delta \tau=1 / B_{w}$ and $\Delta v=1 / T_{\text {coh }}$, respectively [37]. Here, $B_{w}$ corresponds to the bandwidth and $T_{c o h}$ is the duration of $s(t)$. Moreover, particle movements are confined in a window of size $\Delta \tau \times \Delta v$ around the detected peak for the cth cluster. Eq. (27) is evaluated using the location values of each particle and the location that gives the best fitness chosen as the globalBest. Having estimated the parameters of each multipath component in the $c$ th cluster, effects of these multipath components are eliminated as in (20) from the array output for a better estimation in remaining clusters. Iterations, $\eta$, continue until convergence is established or a preset number of iterations is reached.

In the end of this section, we also want to present a novel and efficient PSO-based approach to estimate the number of multipath components in each detected multipath cluster. 
As we pointed out at the beginning of this section, starting point of the PSO-CAF technique is the detection of the first dominant cluster. This means that we have a least one signal in that cluster and we should check whether there exist more signals. Therefore, before conducting a PSO search for parameter estimations in the cluster, an iterative computationally efficient PSO search is performed to estimate the number of signals in the cluster. Having estimated the number and parameters of the multipath components same approach is applied for the next detected cluster. To sum up, we added an additional PSO search to each cluster to be performed only in the first PSO-CAF iteration, $\eta=1$. Let $d_{c}$ is the hypothetical number of signals in the $c$ th cluster. In each step, $i$, the detection problem is formulated as testing the hypothesis $\mathcal{H}_{i-1}$ against the alternative $\mathcal{A}_{i}$ :

- $\mathcal{H}_{i-1}: i-1$ signals exist,

- $\mathcal{A}_{i}: i$ or more than $i$ signals exist.

Starting from $i=1$, this test is performed stepwise until the hypothesis $\mathcal{H}_{i-1}$ is accepted. For $i=1$, since a signal has four parameters to be estimated, a $4 \times 1$-dimensional PSO optimization is conducted based on the derivations presented in this section and $\mathcal{A}_{1}$ is automatically selected to check whether there exist only one or more signals. For each following step, $i, 4 \times i$-dimensional PSO optimization is performed and the following test statistic is used:

$\frac{\left.\max \left|\boldsymbol{\chi}_{i^{\prime}}(\tau, v)\right|\right)}{\operatorname{median}(|\boldsymbol{\chi}(\tau, v)|)} \gtrless_{\mathcal{H}_{i-1}}^{\mathcal{A}_{i}} \gamma \quad \forall i^{\prime}, i^{\prime}=1, \ldots, i$,

where $\boldsymbol{\chi}_{i^{\prime}}(\tau, v)=\hat{\zeta}_{i^{\prime}} \hat{\mathbf{A}}\left(\tau, v ; \mathbf{p}_{g, i}\left[\boldsymbol{\varphi}_{i^{\prime}}\right]\right)$ is the calculated CAF for the $i^{\prime}$ th hypothetical signal using the parameters found in the end of fast PSO search for the cluster. $\mathbf{p}_{g, i}$ is the globalBest particle, which is basically the best solution found by the swarm. $\mathbf{p}_{g, i}\left[\boldsymbol{\varphi}_{i^{\prime}}\right]$ is the parameter estimates for the $i^{\prime}$ th hypothetical signal. $\chi(\tau, v)$ is the incoherently integrated CAF, given in (17). Noise level on the incoherently integrated CAF is quantified with the median operator, since it is relatively insensitive to possible outliers. Moreover, $\gamma$ is the properly chosen threshold and can be determined accurately. Some approaches on the selection of the threshold can be found in $[42,49,50]$.

There are some important points that should be emphasized regarding the number of signals estimation using PSO. First of all, this additional PSO search is computationally very efficient since it requires much fewer number of particles and optimization iterations than the parameter estimation procedure. The aim is to detect number of signals that produce a CAF, which should have a acceptable peak level to be decided as a signal source. Therefore, less number of PSO iterations are enough. Moreover, in each source number detection steps, $i$, globalBest position, $\mathbf{p}_{g, i-1}$, is used as a prior information in half of the initial positions of particles that are used in the $i$ th step. In another words, some portion of the half of the particles in the $i$ th step initialized with the best estimates found in the $i-1$ th step. In vectorial form this can be written as $\mathbf{z}_{l / 2, i}=\left[\mathbf{p}_{g, i-1}, \varphi\right]_{1 \times 4 i}$. Second half of the particles are randomly distributed in the search space. Usage of this prior information from the previous step and embedding it to the some particles enables to increase the fitness in the optimization and chance of convergence to global optimum point. Second, parameter estimation performance of the PSO-CAF technique is not effected if more number of signals are estimated since we carry the globalBest information in some particles and these particles will preserve the true parameters for true number of signals. One obvious choice may be to add one to the estimated source number, which will mostly eliminate parameter estimation errors due to the possible missing source number estimations. Lastly, source number estimation performed only once in the first PSO-CAF iteration, $\eta=1$.

\section{Simulation results}

In this section, we present results of simulated experiments conducted to compare the performances of the PSO-CAF, SAGE and PSO-ML techniques on signals at different SNR values. The Cramer-Rao lower bound (CRLB) for the joint estimation problem is also included for comparison. PSO-ML is a recently proposed technique, which applies PSO to ML criterion to estimate the path parameters [52]. Since, PSO-ML does not exploit the delay-Doppler localization of the multipath components, it operates over significantly higher dimensional search space than the PSO-CAF. Note that, in PSO-CAF, PSO is applied to vectorized delay-Doppler patches rather than time-domain signals. Therefore, delay-Doppler diversity of the multipath components is effectively exploited.

In the experiments, received signals of a circular receiver array of $M=9$ omnidirectional sensors at positions [rcos $(m 2 \pi / M), r \sin (m 2 \pi / M)], \quad 1 \leq m \leq M$, is simulated. The radius of the array $r=\lambda / 4 \sin (\pi / M)$ is chosen such that the distance between two neighboring sensors is $\lambda / 2$, where $\lambda$ is the carrier wavelength. The transmitted training signal consists of six Barker-13 coded pulses with a duration of $13 \Delta \tau$ where $\Delta \tau$ is the chip duration. The pulse repetition interval is $30 \Delta \tau$ resulting a total signal duration of $q T=$ $167 \Delta \tau$. The SNR is defined at a single sensor relative to the noise variance as $E\left[\left|x_{m}(t)\right|^{2}\right] / E\left[\left|n_{m}(t)\right|^{2}\right]$. The joint root mean squared error (rMSE), the basis of our comparisons, is defined for each of the multipath parameters $\left(\beta_{i}: \theta_{i}, \phi_{i}\right.$, $\left.\tau_{i}, v_{i}, i=1, \ldots, d\right)$ as

$\operatorname{rMSE}_{\boldsymbol{\beta}}=\sqrt{\frac{1}{d N_{r}} \sum_{\mu=1}^{N_{r}} \sum_{i=1}^{d}\left[\hat{\beta}_{i}^{\mu}-\beta_{i}^{\mu}\right]^{2},}$

where $N_{r}$ is the number of Monte-Carlo simulations, $\hat{\beta}_{i}^{\mu}$ is one of the parameter estimates of the $i$ th signal path found in the $\mu$ th simulation and $\beta_{i}^{\mu}$ is one of the true parameter values of the $i$ th path in the $\mu$ th simulation. Four hundred Monte Carlo realizations are conducted in the experiments and in each realization, discrete true parameter values are randomly perturbed in the very vicinity of themselves. Moreover, for both of the experiments, the same PSO settings, such as swarm size, update rules, swarm topology and swarm initialization, are chosen based on recommendations in the literature and empirical simulations [35]. We observed that fine tuning the parameters would not provide significant improvements. Therefore, here standard PSO is 
Table 2

Ten path parameters. Time-delay, Doppler and complex scaling factor values are normalized by $\Delta \tau, \Delta v$ and $e^{j \psi_{i}}$, respectively. $\psi_{i}$ 's, $i=1, \ldots, d$ are uniformly distributed between $[0,2 \pi]$.

\begin{tabular}{llllll}
\hline Path & $\theta$ (deg.) & $\phi$ (deg.) & $\tau / \Delta \tau$ & $v / \Delta v$ & $\zeta / e^{j \psi_{i}}$ \\
\hline 1 & 45 & 25 & 1.16 & 1.1 & 1 \\
2 & 50 & 35 & 1.41 & 1.4 & 0.9 \\
3 & 55 & 40 & 1.16 & 2.6 & 0.9 \\
4 & 60 & 45 & 1.41 & 2.9 & 1 \\
5 & 65 & 50 & 3.08 & 2.9 & 0.9 \\
6 & 70 & 55 & 3.33 & 2.6 & 0.85 \\
7 & 75 & 38 & 3.16 & 1.4 & 1 \\
8 & 57 & 47 & 3.5 & 1.1 & 0.8 \\
9 & 63 & 43 & 4.41 & 2.1 & 0.9 \\
10 & 68 & 33 & 4.66 & 2.3 & 0.92 \\
\hline
\end{tabular}

a
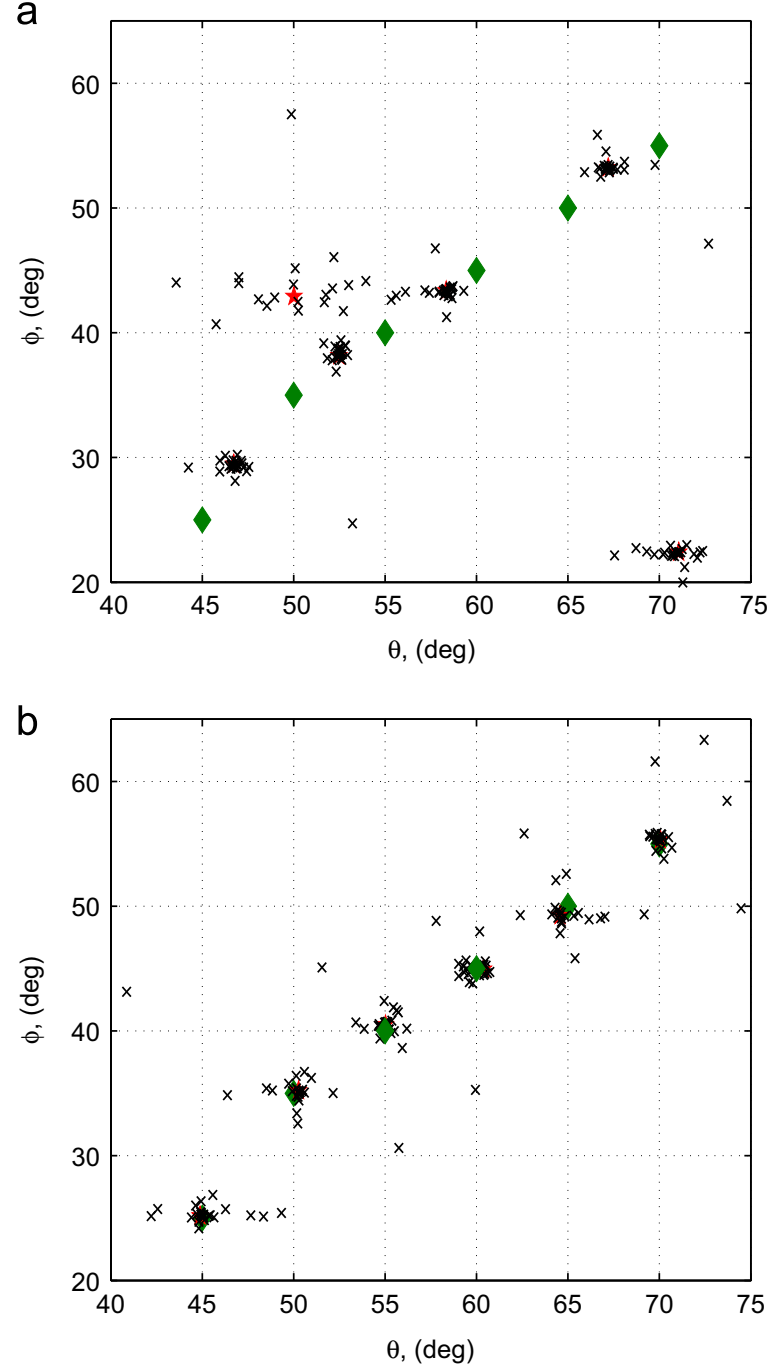

Fig. 5. One snapshot coordinates, obtained by using the PSO-CAF, of particles $(\mathbf{z}, \times)$, exact path parameter values $(\bullet)$ and globalBest $\left(\mathbf{p}_{g}, \star\right)$ distributed on the azimuth $(\theta)$-elevation $(\phi)$ plane. (a) No clustering, PSO is conducted in 24-dimensional space. (b) Three clusters, parallel PSO is conducted in each of them in eight-dimensional spaces. used and results of different PSO variants are not presented. Initial locations and velocities of the particles are randomly distributed throughout the search space. As stated previously, size of the delay-Doppler swarm search space is taken as $\Delta \tau \times \Delta v$ around the detected peak of each cluster. Number of particles in the swarm is chosen as 50 . Necessary number of PSO evaluations and SAGE iterations are conducted for PSO-ML, PSO-CAF and SAGE techniques, respectively, to ensure the convergence.

In the first experiment, we considered a multipath scenario with six paths, whose parameters are given in the top six rows of Table 2 . Note that these six paths are clustered in three clusters each containing two paths. Moreover, multipaths in each cluster have a separation of lower than $0.5 \Delta \tau$ in delay and $0.5 \Delta v$ in Doppler,
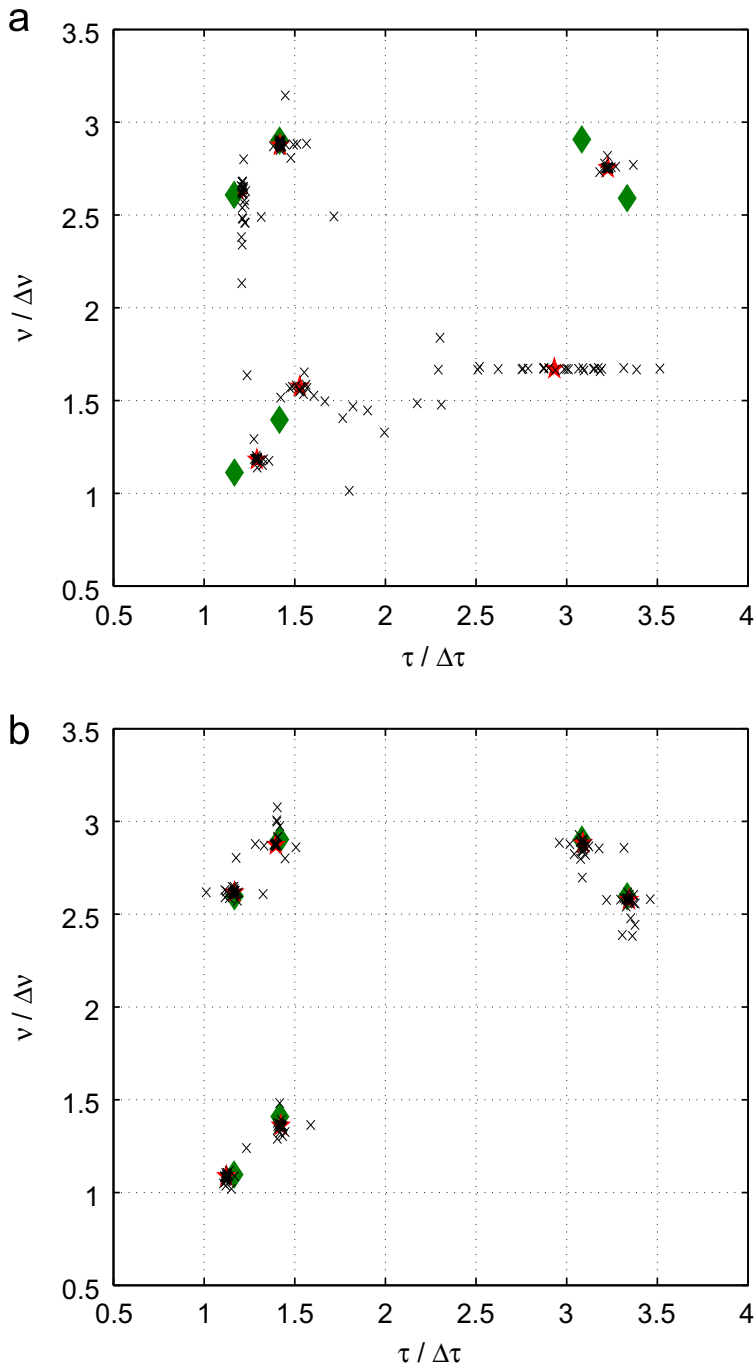

Fig. 6. One snapshot coordinates, obtained by using the PSO-CAF, of particles $(\mathbf{z}, \times)$, exact path parameter values $(\bullet)$ and globalBest $\left(\mathbf{p}_{g}, \star\right)$ distributed on the delay-Doppler plane. (a) No clustering, PSO is conducted in 24-dimensional space. (b) Three clusters, parallel PSO is conducted in each of them in eight-dimensional spaces. 
which make the estimation procedure hard. As stated previously and presented in Fig. 1(a) and (b), the key advantage of the PSO-CAF technique is the localization of different multipath signals to their respective delay and Doppler cells by transforming the array signal outputs to the CAF domain. By this way, we are able to use PSO in lower dimensional parameter search spaces in each cluster to estimate the respective path parameters. The performance improvement due to clustering on delay-Doppler domain is presented in Figs. 5 and 6 for the PSO-CAF technique. In the figures one snapshot coordinates of particles $(\mathbf{z}, \times)$, exact path parameter values $(\bullet)$ and coordinate of globalBest $\left(\mathbf{p}_{g}, \star\right)$ are plotted during the PSO optimization. As can be seen, when all the paths are tried to be identified without delay-Doppler domain clustering, particles typically converge to local minima of the fitness function and rarely reach the exact path parameter coordinates. However, if we conduct three separate eight-dimensional PSO path parameter searches on each cluster, particles converge to the global minima in each cluster in a shorter time with increased frequency. In Fig. 7(a), normalized fitness progress curves of PSO-ML and PSO-CAF techniques are seen. As expected, PSO-CAF has better convergence properties. Fig. 7(b) shows the normalized error progress of the array output estimates of the SAGE algorithm. All simulations are conducted on an HP xw6400 Workstation with Intel Xeon $3 \mathrm{GHz}$ processor. A single iteration for the PSO-CAF, the PSO-ML and the SAGE techniques take approximately as $2.5,1.1$, and $9.4 \mathrm{~s}$, respectively. As shown in Fig. 7(a) and (b), the PSO-CAF,
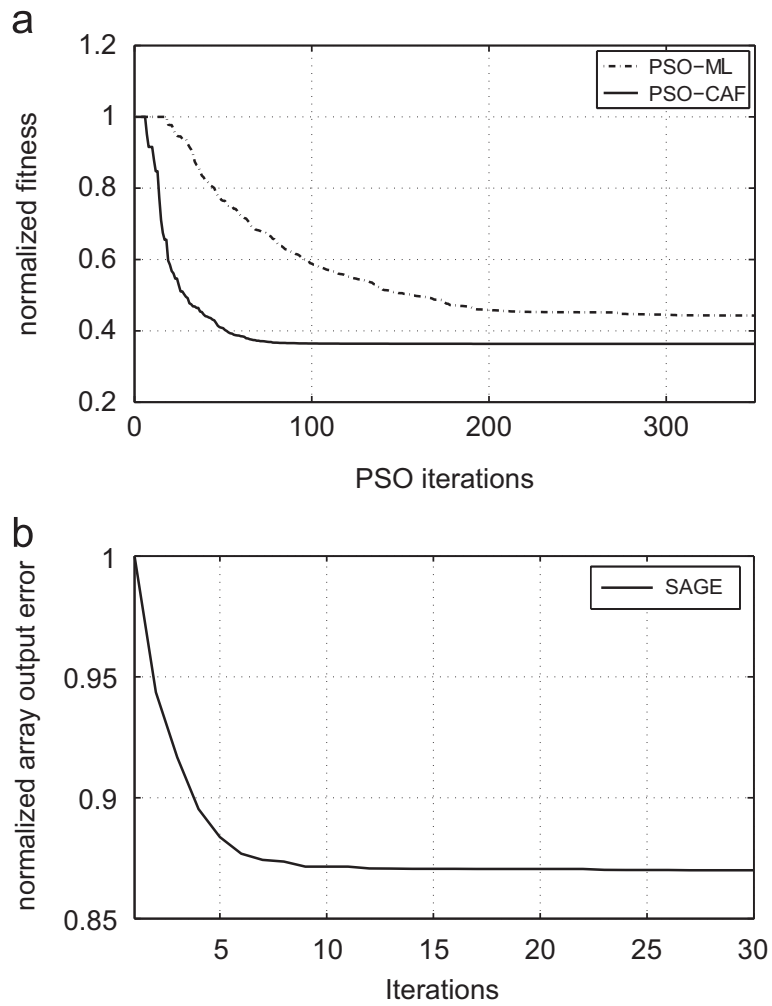

Fig. 7. (a) Normalized fitness progress curves of the PSO-ML and the PSO-CAF. (b) Normalized array output error progress curve of SAGE. the PSO-ML and the SAGE techniques establish their convergence at around 80, 200 and 10 iterations. Therefore, until convergence, the PSO-CAF, the PSO-ML and the SAGE techniques require approximately 200, 220, and $94 \mathrm{~s}$, respectively. Since the PSO based techniques can be implemented on a multicore processor environment with significantly less interprocessor communication requirements, the processing times can be reduced to the
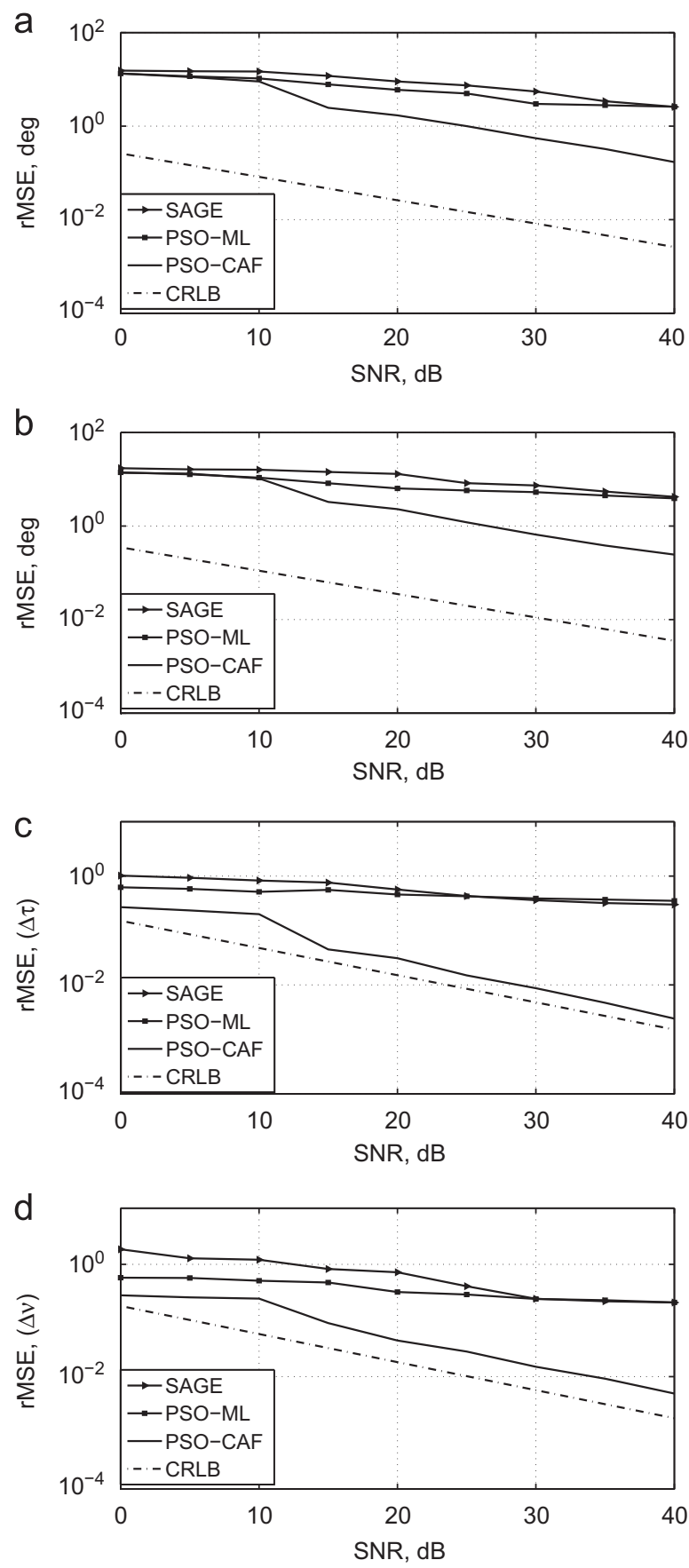

Fig. 8. Joint-rMSE, obtained with the PSO-CAF, the PSO-ML and the SAGE, of (a) azimuth, (b) elevation, (c) time-delay and (d) Doppler shift of six signal paths. Dash-dot line represents the CRLB. 
level of the SAGE technique. Therefore, in the following we will base our comparison results to the accuracy of the estimated parameters. Fig. 8 shows the joint rMSE values obtained from the SAGE, PSO-ML and PSO-CAF for various SNR values. Also to provide a lower bound on the error, CRLB is included. Obtained results shows the superior performance of the PSO-CAF over the PSO-ML and the SAGE techniques for all SNR values. The PSO-ML and the SAGE techniques have similar performances at high SNR values, however, at lower SNR values the PSO-ML outperforms the SAGE technique. Moreover, histograms of joint rMSE of each technique are presented in Fig. 9 to provide an insight into the failure statistics. Consistent with the plots, most of the time, the PSO-ML and SAGE techniques convergence to local points. As stated previously, multipaths in each cluster are localized very closely in delay-Doppler space. Therefore, even for a one cluster scenario having two multipaths, where there is no need for a clustering, the SAGE will fail to converge to global point due to the relative very close position of multipaths on delay-Doppler domain. By using (4) and (5), in Fig. 10, estimated $\widehat{S N R}_{P S O-C A F} / \widehat{S N R}_{P S O-M L}$ ratio is plotted for threshold and asymptotic regions of estimation performance. The PSO-CAF combines diversity better than the PSO-ML which enable detector to accurately retrieve the transmitted information.

In the second experiment, we considered a multipath scenario where there exist five clusters containing two paths each totaling 10 paths with parameters tabulated in Table 2, distributed in five different clusters. To clarify the detection process of delay-Doppler cells corresponding to each multipath cluster, locations of five different clusters on the CAF surface are presented at different SNR values in Fig. 11. It is shown that even at the $10 \mathrm{~dB}$ SNR, all clusters are localized and can be identified on the CAF detection surface. Note that the number of paths exceeds the number of sensors which would made it impossible to resolve with narrowband systems. However, if there exist fewer paths than the number of array elements in each resolvable delay-Doppler cell then delay-Doppler domain diversity of the paths can be exploited to resolve the paths in wideband communication systems. Fig. 12 illustrates the joint rMSE values obtained from SAGE, PSO-ML and PSO-CAF for various SNR values. Similar to the results of the first experiment, PSO-CAF is able to resolve multipath

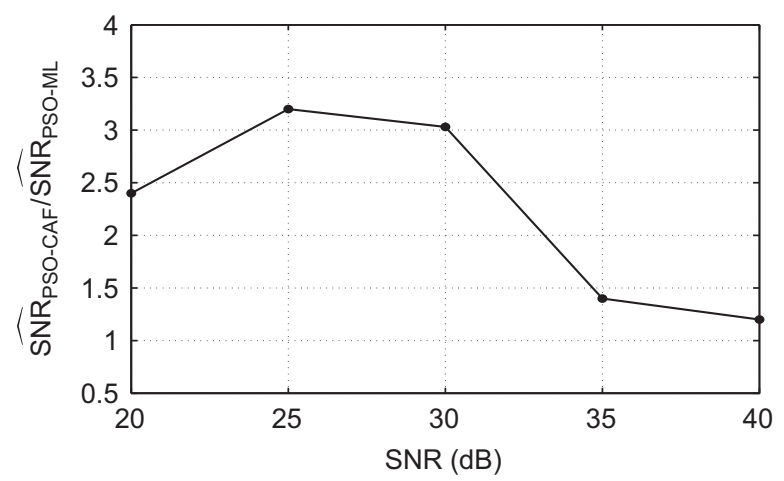

Fig. 10. Ratio of estimated SNR levels of the PSO-CAF and the PSO-ML techniques for threshold and asymptotic regions of estimation performance.

\section{a}

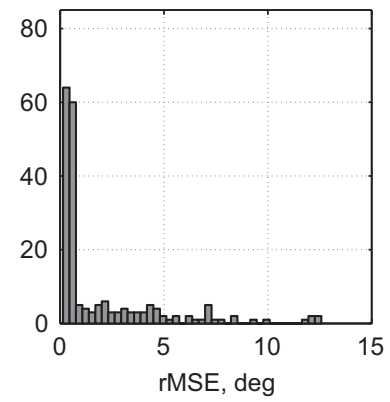

e

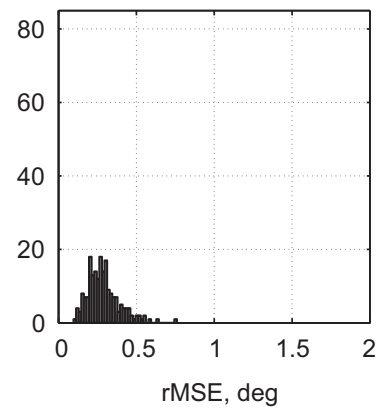

b

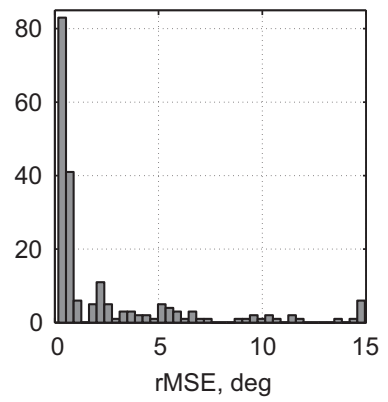

f

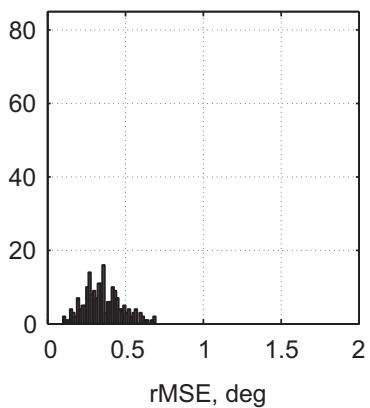

C

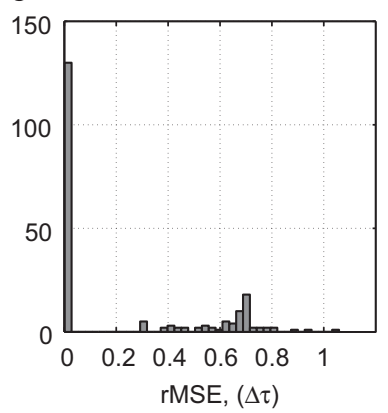

g

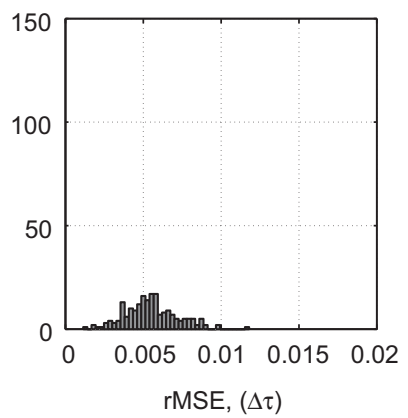

d

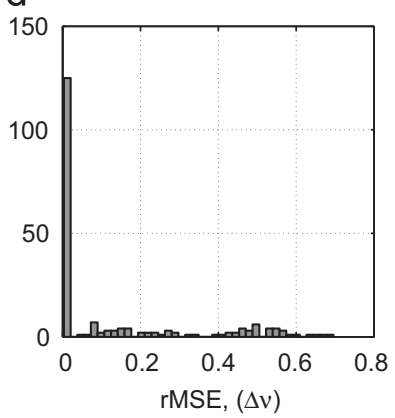

h

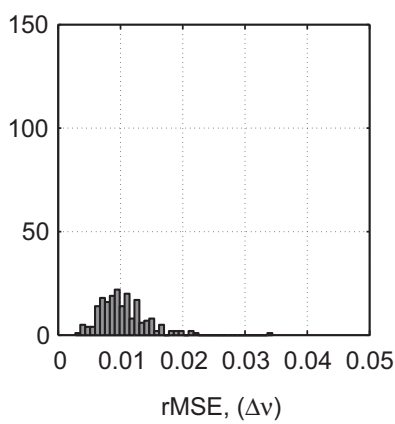

Fig. 9. Histograms of joint rMSE values of azimuth, elevation, delay and Doppler estimates obtained with the PSO-ML and PSO-CAF techniques. (a-d) PSO-ML and (e-h) PSO-CAF. 

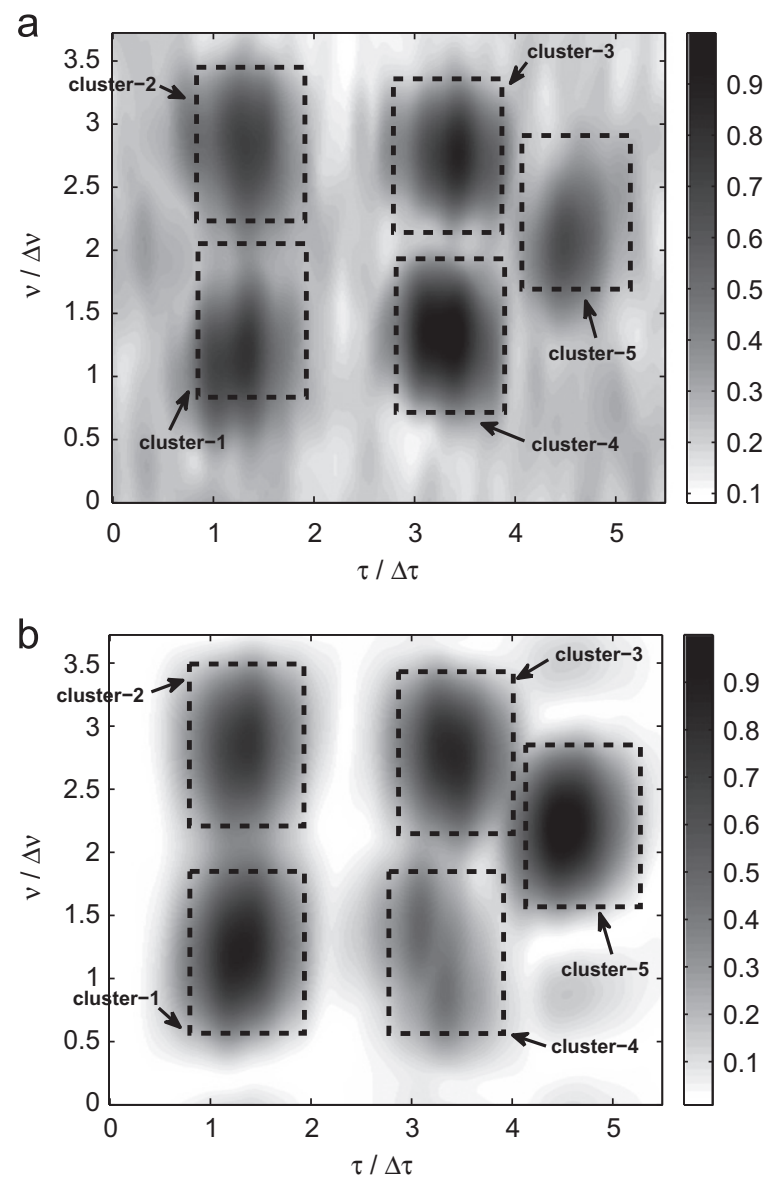

Fig. 11. CAF's between received signal, consisting of 10 multipath components, with the transmitted signal at (a) $10 \mathrm{~dB}$ and (b) $35 \mathrm{~dB}$.

components even in this scenario successfully and outperforms the PSO-ML and SAGE.

In the third experiment, we will provide simulation results of the proposed PSO-based source number estimation technique. Same multipath scenario is used as in the first experiment. However, now much less number of particles and optimization iterations are allocated. Number of particles is chosen as 15 and 40 optimization iterations are conducted. As we have noticed before, since allocated resources for swarm optimization are low and number of signal detection is performed only once in the first PSO-CAF iteration, this process does not effect the overall computational time. Two hundred Monte Carlo simulations are conducted. Probability of correct source number detection for different SNR values is seen in Fig. 13. For most of the SNR values, almost always correct source number is detected. Moreover, it is important to point out that, for SNR values between 15 and $45 \mathrm{~dB}$, very low probability of false detections $(\sim 0.03)$ is occurred. The reason was the overestimation of source number not the underestimation. Therefore, as we have pointed out that earlier, this small false detection rate will not effect the parameter estimation performance of the proposed technique.
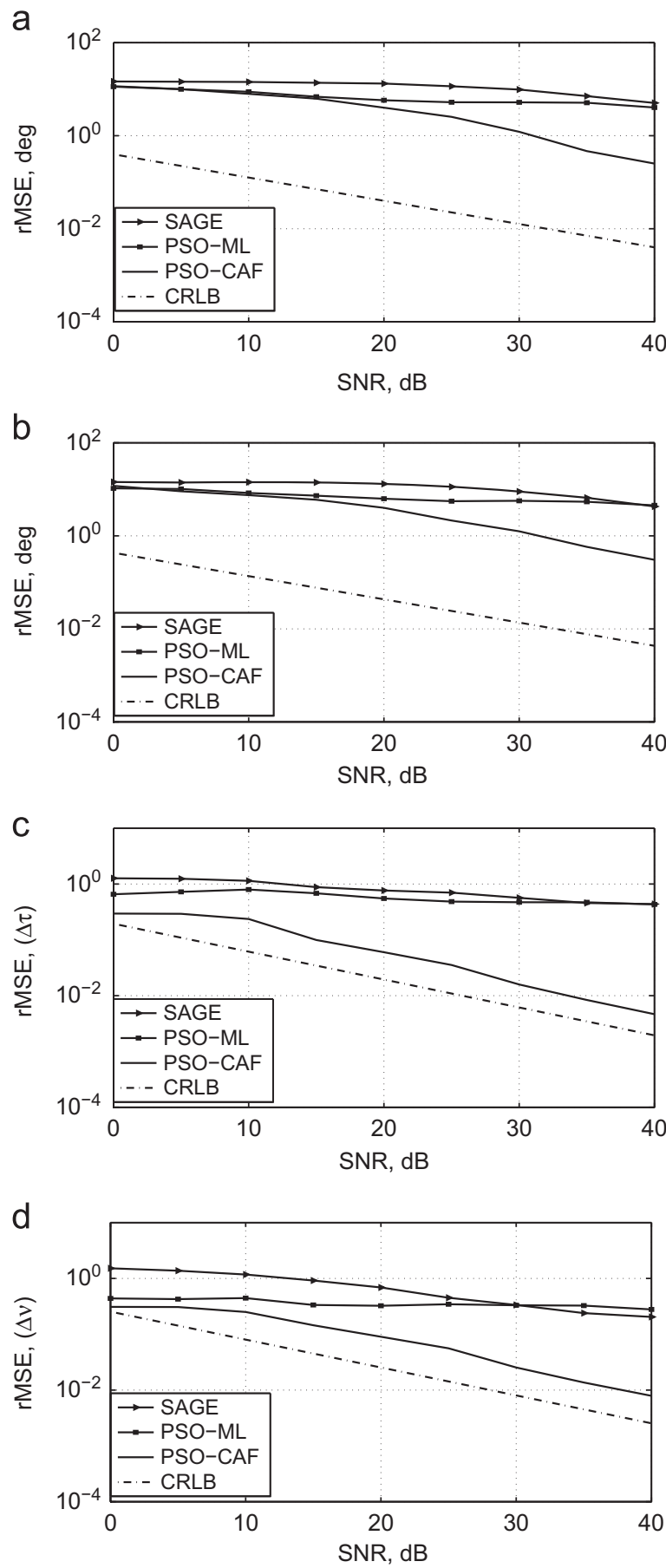

Fig. 12. Joint-rMSE, obtained with the PSO-CAF, the PSO-ML and the SAGE, of (a) azimuth, (b) elevation, (c) time-delay and (d) Doppler shift of 10 signal paths. Dash-dot line represents the CRLB.

\section{Conclusions}

A new multipath channel parameter estimation technique called the PSO-CAF is proposed. PSO-CAF transforms the received array outputs to delay-Doppler domain by CAF calculation for efficient exploration of the delay-Doppler diversity of the multipath signal components. Clusters of 


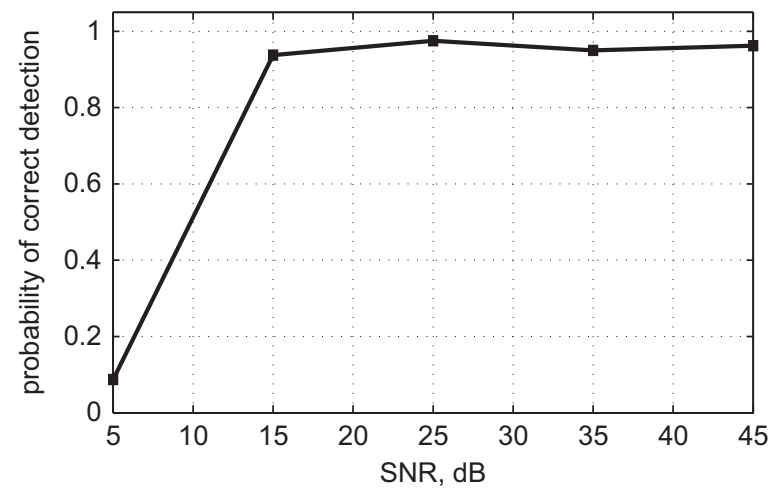

Fig. 13. Correct source number detection probabilities obtained by the PSO-based source number estimation technique for different SNR values.

multipath components are identified in the delay-Doppler domain. Localization of multipath components to their respective delay and Doppler cells enabled the reformulation of the channel identification problem as a set of loosely coupled optimization problems in lower dimensional parameter spaces. PSO is used to identify parameters of multipath components in each cluster. Simulation results show that the PSO-CAF provides significantly better parameter estimates than the SAGE and recently proposed PSO-ML technique.

\section{References}

[1] H. Krim, M. Viberg, Two decades of array signal processing research: the parametric approach, IEEE Signal Process. Mag. 13 (4) (1996) 67-94.

[2] A.P. Dempster, N.M. Laird, D.B. Rubin, Maximum likelihood from incomplete data via the EM algorithm, J. R. Stat. Soc. 39 (1) (1977) $1-38$.

[3] J.A. Fessler, A.O. Hero, Space-alternating generalized expectationmaximization algorithm, IEEE Trans. Signal Process. 42 (10) (1994) 2664-2677.

[4] P.J. Chung, J.F. Bohme, DOA estimation using fast EM and SAGE algorithms, Signal Process. 82 (2002) 1753-1762.

[5] N. Czink, X. Yin, H. Ozcelik, M. Herdin, E. Bonek, B.H. Fleury, Cluster characteristics in a MIMO indoor propagation environment, IEEE Trans. Wireless Commun. 6 (4) (2007) 1465-1475.

[6] A. Kocian, I. Land, B.H. Fleury, Joint channel estimation, partial successive interference cancellation, and data decoding for DSCDMA based on the SAGE algorithm, IEEE Trans. Commun. 55 (6) (2007) 1231-1241.

[7] B.H. Fleury, M. Tschudin, R. Heddergott, D. Dahlhaus, K.I. Pedersen, Channel parameter estimation in mobile radio environments using the SAGE algorithm, IEEE J. Sel. Areas Commun. 17 (3) (1999) 434-450.

[8] X. Fu, H. Minn, C.D. Cantrell, Two novel iterative joint frequencyoffset and channel estimation methods for OFDMA uplink, IEEE Trans. Commun. 56 (3) (2008) 474-484.

[9] F. Yang, J. Song, Y. Zhang, C. Pan, Z. Yang, SAGE-based estimation of doubly selective channel with an orthogonal polynomial model, Signal Process. 88 (4) (2008) 1061-1068.

[10] P.J. Chung, J.F. Bohme, Recursive EM and SAGE-inspired algorithms with application to DOA estimation, IEEE Trans. Signal Process. 53 (8) (2005) 2664-2677.

[11] M.B. Guldogan, O. Arikan, Particle swarm optimization based channel identification in cross-ambiguity domain, in: IEEE Int. Conf. Acoust. Speech Sign. Processing (ICASSP), 2010.

[12] M.B. Guldogan, O. Arikan, F. Arikan, A new technique for direction of arrival estimation for ionospheric multipath channels, J. Adv. Space Res. 44 (6) (2009) 653-662.

[13] M.B. Guldogan, O. Arikan, Comparison of the CAF-DF and SAGE algorithms in multipath channel parameter estimation, in: IEEE Wksp. Sensor Array and Multichannel Signal Processing (SAM), 2008.
[14] M. Wax, T. Kailath, Detection of signals by information theoretic criteria, IEEE Trans. Acoust. Speech Signal Process. 33 (2) (1985) 387-392.

[15] R.F. Brcich, A.M. Zoubir, P. Pelin, Detection of sources using bootstrap techniques, IEEE Trans. Signal Process. 50 (2) (2002) 206-215.

[16] P.J. Chung J.F. Bohme, C.F. Mecklenbrauker, A.O. Hero, Detection of the number of signals using the Benjamini-Hochberg procedure, IEEE Trans. Signal Process. 55 (6) (2007) 2497-2508.

[17] S. Aouada, A. Zoubir, C. See, A comparative study on source number detection, in: Signal Processing and its Applications, 2003.

[18] A. Goldsmith, Wireless Communications, Cambridge University Press, 2005.

[19] J. Kennedy, R. Eberhart, Particle swarm optimization, in: IEEE Int. Conf. Neural Networks, 1995.

[20] G.K. Venayagamoorthy, W. Zha, Comparison of nonuniform optimal quantizer designs for speech coding with adaptive critics and particle swarm, IEEE Trans. Ind. Appl. 43 (1) (2007) 238-244.

[21] W. Pedrycza, K. Hirota, Fuzzy vector quantization with the particle swarm optimization: a study in fuzzy degranulation information processing, Signal Process. 87 (9) (2007) 2061-2074.

[22] C. Potter, G.K. Venayagamoorthy, K. Kosbar, RNN based MIMO channel prediction, Signal Process. 90 (2) (2010) 440-450.

[23] Y. Chen, V.K. Dubey, Ultra-wideband source localization using a particle-swarm-optimized capon estimator from a frequencydependent channel modeling viewpoint, EURASIP J. Appl. Signal Process. 2005 (12) (2005) 1854-1866.

[24] Y. Yuan, Z. He, M. Chen, Virtual MIMO-based cross-layer design for wireless sensor networks, IEEE Trans. Veh. Technol. 55 (3) (2006) 856-864.

[25] S. Selvan, C. Xavier, N. Karssemeijer, J. Sequeira, R. Cherian, B. Dhala, Parameter estimation in stochastic mammogram model by heuristic optimization techniques, IEEE Trans. Inf. Technol. Biomed. 10 (4) (2006) 685-695.

[26] K. Veeramachaneni, L. Osadciw, P. Varshney, An adaptive multimodal biometric management algorithm, IEEE Trans. Syst. Man Cybern. C 35 (3) (2005) 344-356.

[27] Z.L. Gaing, A particle swarm optimization approach for optimum design of PID controller in AVR system, IEEE Trans. Energy Convers. 19 (2) (2004) 384-391.

[28] J. Heo, K. Lee, R. Garduno-Ramirez, Multiobjective control of power plants using particle swarm optimization techniques, IEEE Trans. Energy Convers. 21 (2) (2006) 552-561.

[29] A. Chatterjee, K. Pulasinghe, K. Watanabe, K. Izumi, A particleswarm-optimized fuzzy-neural network for voice controlled robot systems, IEEE Trans. Ind. Electron. 52 (6) (2005) 1478-1489.

[30] Y. Ma, C. Jiang, Z. Hou, C. Wang, The formulation of the optimal strategies for the electricity producers based on the particle swarm optimization algorithm, IEEE Trans. Power Syst. 21 (4) (2006) 1663-1671.

[31] F. Grimaccia, M. Mussetta, R.E. Zich, Genetical swarm optimization: self-adaptive hybrid evolutionary algorithm for electromagnetics, IEEE Trans. Antennas Propag. 55 (3) (2007) 781-785.

[32] M. Wachowiak, R. Smolikova, Y. Zheng, J. Zurada, A. Elmaghraby, An approach to multimodal biomedical image registration utilizing particle swarm optimization, IEEE Trans. Evol. Comput. 8 (3) (2004) 289-301.

[33] H.Y. Chen, J.J. Leou, Saliency-directed image interpolation using particle swarm optimization, Signal Process. 90 (5) (2010) 1676-1692.

[34] D.E. Goldberg, Genetic Algorithms in Search, Optimization and Machine Learning, Addison-Wesley Longman Publishing, 1989.

[35] D. Bratton, J. Kennedy, Defining a standard for particle swarm optimization, in: IEEE Int. Symp. Swarm Intelligence (SIS), 2007.

[36] P.M. Woodward, Probability and Information Theory with Application to Radar, Pergamon, London, 1953.

[37] N. Levanon, E. Mozeson, Radar Signals, Wiley-IEEE Press, 2004.

[38] L. Cohen, Time Frequency Analysis: Theory and Applications, Prentice Hall, 1995.

[39] H.L.V. Trees, Detection, Estimation and Modulation Theory, Part III: Radar-Sonar Signal Processing and Gaussian Signals in Noise, John Wiley, 2001.

[40] B.R. Mahafza, MATLAB Simulations for Radar Systems Design, Chapman and Hall/CRC, 2003.

[41] P. Flandrin, A time-frequency formulation of optimum detection, IEEE Trans. Acoust. Speech Signal Process. 36 (10) (1988) 1377-1384.

[42] C. Candan, On the optimality of detectors defined over the ambiguity plane, in: IEEE Radar Conf., 2009.

[43] R.H. Barker, Group synchronizing of binary digital sequences, Commun. Theory (1953) 273-287. 
[44] S. Golomb, R. Scholtz, Generalized Barker sequences, IEEE Trans. Inf. Theory 11 (4) (1965) 533-537.

[45] A. Saleh, R. Valenzuela, A statistical model for indoor multipath propagation, IEEE J. Sel. Areas Commun. (1987) 128-137.

[46] Q.H. Spencer, B.D. Jeffs, M.A. Jensen, A.L. Swindlehurst, Modeling the statistical time and angle of arrival characteristics of an indoor multipath channel, IEEE J. Sel. Areas Commun. 18 (3) (2000) 347-360.

[47] W.U. Bajwa, J. Haupt, A.M. Sayeed, R. Nowak, Compressed channel sensing: a new approach to estimating sparse multipath channels, Proc. IEEE 98 (6) (2010) 1058-1076.

[48] M.I. Skolnik, Introduction to Radar Systems, McGraw-Hill, 2001.
[49] G.T. Zhou, M. Ikram, Unsupervised detection and parameter estimation of multi-component sinusoidal signals in noise, in: Asimolar Conf. on Sig., Sys. and Computers, 2000.

[50] H. Hindberg, A. Hanssen, S. Olhede, Thresholding the ambiguity function, in: IEEE Int. Conf. Acoust. Speech Sign. Processing (ICASSP), 2008

[51] M.B. Guldogan, O. Arikan, A novel array signal processing technique for multipath channel parameter estimation, in: IEEE Signal Process. and Commun. Applications (SIU), 2007.

[52] M. Li, Y. Lu, Maximum likelihood DOA estimation in unknown colored noise fields, IEEE Trans. Aerosp. Electron. Syst. 44 (3) (2008) 1079-1090. 